\title{
Endoscopic mucosal resection for treatment of early gastric cancer
}

H Ono, H Kondo, T Gotoda, K Shirao, H Yamaguchi, D Saito, K Hosokawa, T Shimoda, $S$ Yoshida

\begin{abstract}
Background-In Japan, endoscopic mucosal resection (EMR) is accepted as a treatment option for cases of early gastric cancer (EGC) where the probability of lymph node metastasis is low. The results of EMR for EGC at the National Cancer Center Hospital, Tokyo, over a 11 year period are presented.
\end{abstract}

Methods-EMR was applied to patients with early cancers up to $30 \mathrm{~mm}$ in diameter that were of a well or moderately histologically differentiated type, and were superficially elevated and/or depressed (types I, IIa, and IIc) but without ulceration or definite signs of submucosal invasion. The resected specimens were carefully examined by serial sections at $2 \mathrm{~mm}$ intervals, and if histopathology revealed submucosal invasion and/or vessel involvement or if the resection margin was not clear, surgery was recommended. Results-Four hundred and seventy nine cancers in 445 patients were treated by EMR from 1987 to 1998 but submucosal invasion was found on subsequent pathological examination in 74 tumours. Sixty nine percent of intramucosal cancers $(278$ I 405) were resected with a clear margin. Of 127 cancers without "complete resection", 14 underwent an additional operation and nine were treated endoscopically; the remainder had intensive follow up. Local recurrence in the stomach occurred in 17 lesions followed conservatively, in one lesion treated endoscopically, and in five lesions with complete resection. All tumours were diagnosed by follow up endoscopy and subsequently treated by surgery. There were no gastric cancer related deaths during a median follow up period of 38 months (3-120 months). Bleeding and perforation (5\%) were two major complications of EMR but there were no treatment related deaths.

Conclusion-In our experience, EMR allows us to perform less invasive treatment without sacrificing the possibility of cure. (Gut 2001;48:225-229)

Keywords: endoscopic mucosal resection; early gastric cancer

Dr H Kondo, Department of Endoscopy and

Gastrointestinal Oncology,

National Cancer Center

Hospital, 5-1-1 Tsukiji,

Chuo-ku, Tokyo 104-0045,

Japan.htkondoh@ncc.go.jp

Accepted for publication 18 July 2000

Early gastric cancer (EGC) is defined as that confined to the mucosa or submucosa, regardless of the presence or absence of regional lymph node metastasis. It has been increasing in Japan, ${ }^{1}$ accounting for approximately $60 \%$ of all resected cases in our institution. In Japan, the five year survival rate of patients with EGC is more than $90 \%$ after gastrectomy with complete removal of primary and secondary lymph nodes. ${ }^{2}{ }^{3}$ The incidence of nodal metastasis of intramucosal and submucosal EGC has been reported as $3 \%$ and $20 \%$, respectively, ${ }^{4}$ and therefore major surgery may be inappropriate in many of these patients. It has been shown that lymphatic vessel invasion, histological ulceration of the tumour, and tumour diameter $(>30 \mathrm{~mm})$ are independent risk factors for regional lymph node metastasis, and in the absence of these risk factors the incidence of lymph node involvement in patients with intramucosal EGC is $0.36 \% .^{5}$ These patients may be appropriate for endoscopic mucosal resection (EMR).

Fifteen years have passed since EMR was introduced in Japan. ${ }^{6}$ However, there are still unsolved problems with regard to its indications, techniques, evaluation of curability, and follow up. In this paper, we present the results of EMR for EGC at the National Cancer Center Hospital (NCCH) over an 11 year period.

\section{Patients and methods}

A prospective analysis of 479 EGCs in 445 patients resected by EMR at NCCH, Tokyo, from March 1987 to December 1998 was performed. These were 413 solitary, 17 double, one triple, one quadruple, and 13 metachronous tumours.

Mean age of the patients was 67.6 years (range 34-92), the male to female ratio was $4.13(357 / 88)$, and the follow up period was 3-125 months (median 45 months).

The Japanese Classification of Gastric Carcinoma was used for tumour description. ${ }^{1}$ The indications for EMR are determined by the risk of lymph node metastasis ${ }^{5}$ and such technical considerations as the size and position of the lesion, and the equipment and expertise available locally. Current indications for EMR at the NCCH are shown in table 1. When EMR was introduced to the NCCH in 1987, the indication size was $15 \mathrm{~mm}$ or less in diameter, and multifragment resection was not accepted. However, it was increased to $30 \mathrm{~mm}$ because multifragment resection was introduced based on a review of resected cancers and the development of appropriate endoscopic techniques.
Abbreviations used in this paper: EGC, early gastric cancer; EMR, endoscopic mucosal resection; $\mathrm{NCCH}$, National Cancer Center Hospital. 
Table 1 Indication criteria for endoscopic mucosal resection

Early gastric cancer meeting all of the following.

(1) Well or moderately differentiated type adenocarcinoma

(2) Superficial, elevated, or depressed macroscopic appearance (types I, IIa, IIc)

(3) No ulceration

(4) Diameter $<30 \mathrm{~mm}$

(5) No apparent invasive findings

As it is sometimes difficult to accurately assess the depth of invasion prior to EMR, ${ }^{7}$ we are prepared to remove any lesion that appears to be confined to the mucosa if it meets the other criteria.

In this series, most EMRs were performed by the so called "strip biopsy method", a relatively simple technique that has been described elsewhere. ${ }^{68}$ Since 1997, a new EMR procedure using an insulation tipped diathermic knife (IT knife) was used in the majority of $\operatorname{cases}^{9}$ (fig 1).

Follow up endoscopy was performed at three and six months after EMR in the first year, and yearly thereafter to diagnose local recurrence.

EVALUATION OF RESECTED SPECIMENS AND RESECTABILITY

The resectability of EMR specimens was carefully evaluated both endoscopically and histopathologically in slices at $2 \mathrm{~mm}$ intervals according to the Japanese Classification of Gastric Carcinoma . ${ }^{10}$ After resection in multiple fragments, resectability was evaluated based on completely reconstructed specimens. The current definition of "resection EA", ${ }^{10}$ which means a high probability of cure, is demonstrated in table 2. As submucosal
Table 2 Evaluation of resected specimens by endoscopic mucosal resection The following must be confirmed histologically for "complete

resection":
(1) Intramucosal cancer

(2) Well or moderately differentiated type adenocarcinoma

(3) No histological ulceration

(4) No lymphatic or venous invasion

(5) No tumour invasion to the lateral margin

invasion and/or vessel involvement are regarded as high risks of positive nodes or distant metastasis, surgical intervention was strongly recommended.

Evaluation of invasion to the lateral margin was classified into the following three groups using endoscopic and histopathological evidence:

(1) Complete resection: when the lateral margin was clear endoscopically and pathologically (minimum probability of local recurrence).

(2) Incomplete resection: when the tumour definitely invaded to the lateral margin endoscopically and pathologically (high probability of local recurrence).

(3) Not evaluable: when the tumour was removed endoscopically but its lateral margin was not pathologically evaluable due to a burn effect (burned by diathermic treatment) or mechanical damage, or when reconstruction was difficult due to multifragment resection.

STATISTICAL ANALYSIS

The SAS program (SAS Institute Inc., Cary, North Carolina, USA) was used for all analyses; $p<0.05$ was taken as significant.
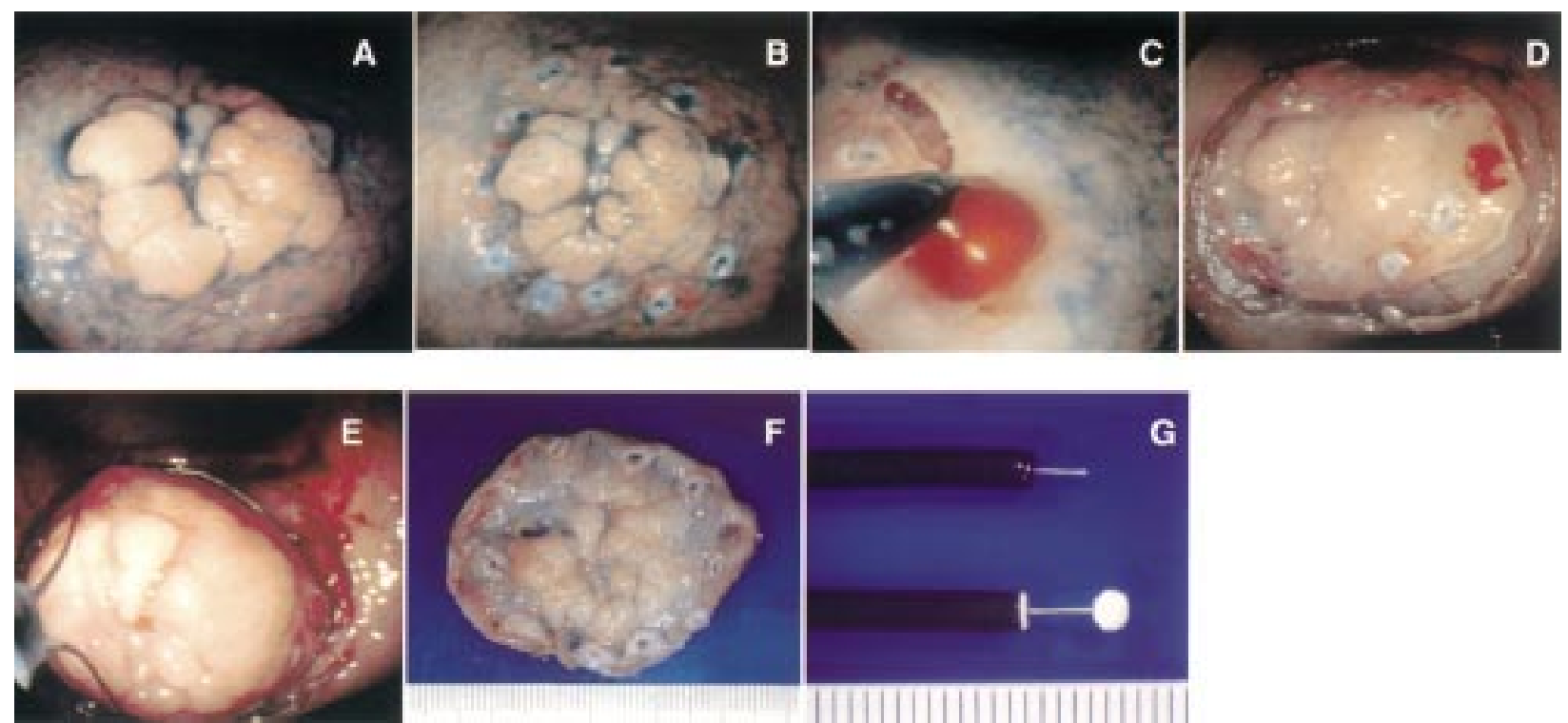

Figure 1 Endoscopic mucosal resection procedure using an IT knife. (A) Superficial elevated (IIa type) early gastric cancer (EGC) located on the lesser curvature of the lower body after spraying with indigo carmine dye. (B) Marking dots were made using a precut knife on the circumference of the target lesion to clarify the margin. (C) After injection of saline with epinephrine $(0.025 \mathrm{mg} / \mathrm{ml})$ into the submucosal layer, an initial cut was made with a conventional needle knife outside of the dots. The IT knife was inserted into this cut and operated to cut around the lesion. (D) The tumour marked by dots was separated from the surrounding normal mucosa. (E) The tumour was removed by standard polypectomy with a combination of cutting and coagulation current in a single fragment. (F) The resected specimen showed well differentiated adenocarcinoma (20×25 mm) with a clear lateral margin. (G) The specifications of the insulation tipped diathermic knife, which was developed by Dr Hosokawa in 1994. The knife consists of a conventional diathermic needle knife (KD-1L; Olympus, fapan) with a ceramic ball at the top to minimise the risk of perforation. 
Table 3 Macroscopic features of the resected tumours

\begin{tabular}{lcrrrrr}
\hline & \multicolumn{7}{c}{ Tumour size (mm) } \\
\cline { 3 - 7 } Type & Mean & \multicolumn{1}{c}{-10} & $11-20$ & $21-30$ & $31-$ & Total \\
\hline 0-I type (protruded) & 19.7 & 5 & 5 & 3 & 2 & 15 \\
0-IIa type (superficial elevated) & 15.7 & 72 & 84 & 21 & 7 & 184 \\
0-IIc type (superficial depressed) & 11.2 & 186 & 77 & 14 & 3 & 280 \\
Total & & 263 & 166 & 38 & 12 & 479 \\
\hline
\end{tabular}

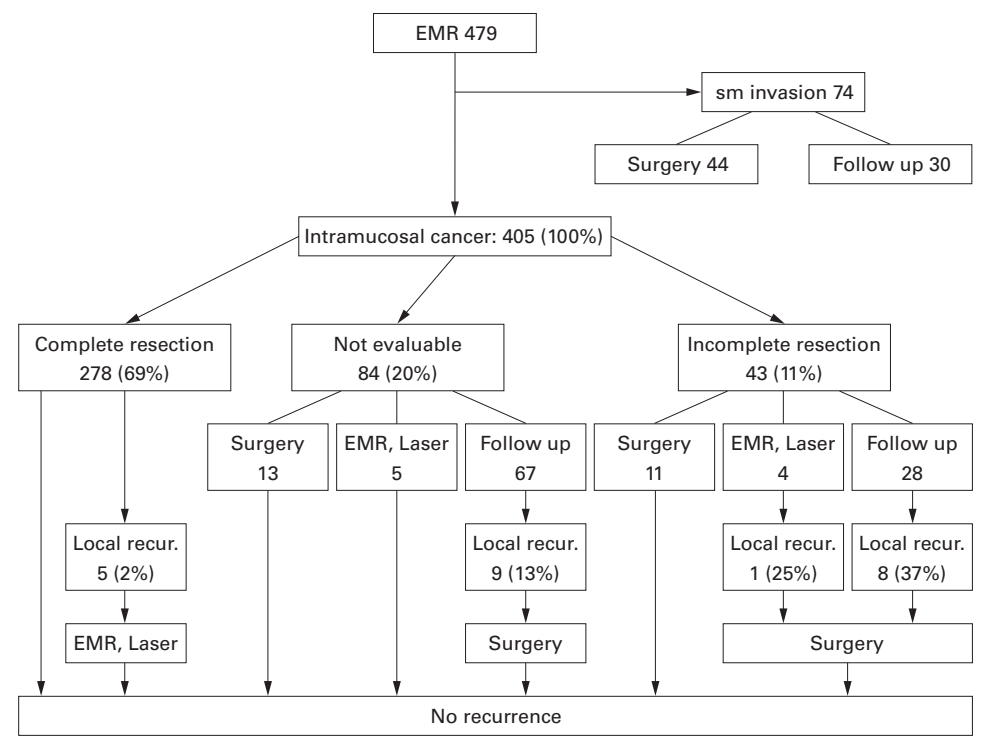

Figure 2 Clinical courses after endoscopic mucosal resection (EMR) for early gastric cancer.

Table 4 Tumour size and invasion to the resection margin of intramucosal cancer

\begin{tabular}{lccccc}
\hline \multicolumn{5}{c}{ Tumour size $(\mathrm{mm})$} \\
\cline { 2 - 6 } & -10 & $11-20$ & $21-30$ & $31-$ & Total \\
\hline Complete resection & $170(72 \%)$ & $86(67 \%)$ & $19(58 \%)$ & $3(38 \%)$ & $278(69 \%)$ \\
Incomplete resection & $21(9 \%)$ & $16(13 \%)$ & $2(6 \%)$ & $4(50 \%)$ & $43(11 \%)$ \\
Not evaluable & $45(19 \%)$ & $26(20 \%)$ & $12(36 \%)$ & $1(13 \%)$ & $84(21 \%)$ \\
Total & $236(100 \%)$ & $128(100 \%)$ & $33(100 \%)$ & $8(100 \%)$ & $405(100 \%)$ \\
\hline
\end{tabular}

Table 5 Tumour location and invasion to the resection margin of intramucosal cancer

\begin{tabular}{|c|c|c|c|c|}
\hline & \multicolumn{3}{|c|}{ Location in the stomach } & \multirow[b]{2}{*}{ Total } \\
\hline & Lower & Middle & Upper & \\
\hline Complete resection & $151(75 \%)$ & $94(65 \%)$ & $33(57 \%)$ & $278(69 \%)$ \\
\hline Incomplete resection & $20(10 \%)$ & $17(12 \%)$ & $6(10 \%)$ & $43(11 \%)$ \\
\hline Not evaluable & $31(15 \%)$ & $34(23 \%)$ & $19(33 \%)$ & $84(21 \%)$ \\
\hline Total & $202(100 \%)$ & $145(100 \%)$ & $58(100 \%)$ & $405(100 \%)$ \\
\hline
\end{tabular}

\section{Results}

EVALUATION OF RESECTED SPECIMENS

The macroscopic appearances of the resected tumours are summarized in table 3. A total of 429 lesions (90\%) were less than $20 \mathrm{~mm}$ in diameter while 280 lesions $(58 \%)$ were of the superficial depressed type (0-IIc type). The tumours were located in the upper third $(82$ lesions, $17 \%$ ), middle third (166 lesions, $35 \%$ ), and lower third (231 lesions, 48\%) of the stomach.

Submucosal invasion was found in 74 lesions on subsequent pathological examination by serial sections (fig 2). These patients were strongly recommended to undergo additional gastrectomy with D2 lymph node dissection. However, 30 patients were merely followed because of minute submucosal invasion, advanced age, other diseases, or the patient's refusal of an additional operation.

Among 405 intramucosal cancers, 278 $(69 \%)$ were resected with a clear lateral margin (table 4 ). The rate of complete resection was lower when lesions had a diameter exceeding $20 \mathrm{~mm}$ and were situated in the upper third of the stomach (tables 4,5 ). Forty three tumours were incompletely resected as there was definite invasion to the lateral margin endoscopically and histologically. Although EMR appeared to be successful endoscopically, 84 lesions could not be evaluated histologically because of diathermic burn, mechanical damage, or failure to retrieve multiple fragments.

CLINICAL OUTCOMES (FIG 2)

Of 278 lesions which were completely resected, five $(2 \%)$ developed local recurrence, and all were subsequently treated with curative intent. The remainder are disease free at a median follow up period of 38 months (3-120 months).

Of 127 lesions whose "complete resection" was not confirmed, 24 underwent resection and no lymph node metastases were found in these patients, and nine were treated by endoscopic therapy and one developed local recurrence after laser ablation. Among the 95 lesions that were followed conservatively, there were 17 local recurrences $(18 \%)$ after a median follow up of four months (2-12 months). All of these patients with recurrence underwent surgery and remain disease free. There were no treatment related or gastric cancer related deaths.

\section{COMPLICATIONS}

Bleeding and perforation were two major complications of EMR. All bleeding was controlled by endoscopic treatment with ethanol injection, endoscopic clipping (HX5LR-1, Olympus, Japan), and spraying of thrombin solution into the stomach. There were 25 cases of perforation ( $5 \%)$; the first four were converted to open surgery. Since then, all perforations have been successfully treated with endoscopic clipping, intubation of a nasogastric tube, and administration of antibiotics.

\section{Discussion}

The EMR technique has been developed mainly in Japan where there is a high incidence of EGC. It is used infrequently in the West. It is important to carefully evaluate the current status of EMR and promote the appropriate use of this technique around the world. The number of patients undergoing EMR is increasing, with about 500 procedures performed in our hospital over the past 11 years. Considering that it generally takes one hour for resection and one week for admission, EMR is a minimally invasive procedure (a patient undergoing surgical resection is hospitalised for approximately three weeks). Our experience suggests that EMR can provide comparable long term survival rates to traditional therapy, provided adherence to strict inclusion 


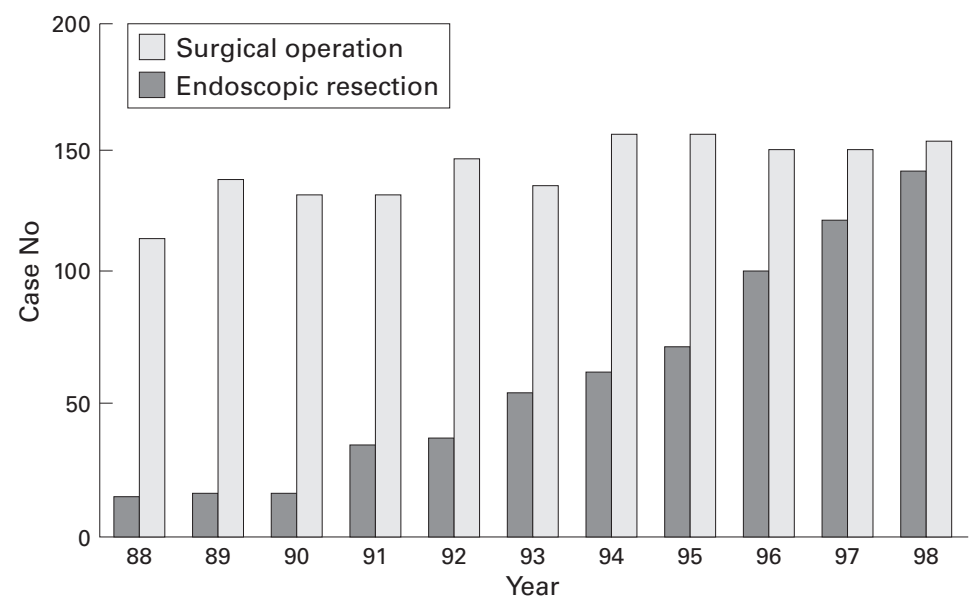

Figure 3 Trends in treatment for early gastric cancer at the National Cancer Center Hospital. of these patients, it may be reasonable to treat patients with minute submucosal invasion by EMR.

In our series, most EMRs were performed by the so called "strip biopsy method". However, we had five local recurrences in "complete resection", all of which had been resected in multiple fragments by this method. Single fragment resection is preferable because with multifragment specimens it is often difficult to reconstruct the entire lesion. We believe it is likely that local recurrence can often be attributed to inappropriate assessment of the multiple fragments of resected specimens.

There have been several reports on endoscopic resection of larger lesions. ${ }^{14}{ }^{15}$ In addition, we have found that the insulation tipped diathermy knife (IT knife) can allow safe resection of a larger lesion in a single piece. The average rate of complete resection increased to more than $90 \%$ and histological evaluation became easier following introduction of this technique.

A follow up programme is important for finding local recurrence and other cancers in the stomach. Thirteen metachronous early cancers were included in our series, which were diagnosed a median period of 23 months after the initial EMR (3-67 months).

The incidence of multiple primary lesions in surgically treated EGC is approximately $10 \%$ at the NCCH. ${ }^{16}$ As EGC is frequently associated with synchronous tumours that may be missed at an initial endoscopic examination, and the stomach may also contain premalignant conditions such as intestinal metaplasia, we should pay attention not only to recurrence but also to other cancers in the stomach.

In summary, based on our experience, it is clear that EMR can provide the same favourable long term survival rates as traditional therapy and is now the treatment of choice for mucosal EGC. We hope to promote its use around the world. the grounds that the cells are of a malignant type and have the potential to invade. ${ }^{11} \mathrm{~A}$ recent analysis showed that the histological type, macroscopic appearance, degree of invasion, and lymph node metastases are the same although many more EGCs are detected in Japan than in the West. ${ }^{12}$ We usually perform EMR for intramucosal cancer using the Japanese criteria, and it is important to have the same diagnostic criteria for gastric neoplastic lesions to propagate EMR around the world.

As it is sometimes difficult to accurately assess the depth of invasion prior to EMR, we remove any lesion without apparent endoscopic findings of submucosal invasion ${ }^{7}$ but $15 \%(74 / 479)$ of all lesions invaded to the submucosa. We do not use endoscopic ultrasonography routinely as it is not sensitive enough to evaluate minute invasion to the submucosa.

Although histological examination revealed minute submucosal invasion in resected EGC, some patients refused to undergo an additional treatment. As no lymph node metastasis was demonstrated in tumours with less than $300 \mu \mathrm{m}$ of submucosal invasion, ${ }^{13}$ and no recurrence has been observed during follow up
We are indebted to Drs M Sasako, T Sano, and H Katai, and K Maruyama for helpful suggestions. The work was supported in part by Grants-in-Aid for Cancer Research and for the 2nd Term Comprehensive 10-year Strategy of Cancer Control from the Ministry of Health and Welfare of Japan Carcinoma. Tokyo: Kanehara and Co., Ltd, 1995.

2 Okamura $\mathrm{T}$, Tsujitani S, Korenaga $\mathrm{D}$, et al. Lymphadenectomy for cure in patients with early gastric cancer and lymph node metastasis. Am $\mathcal{F}$ Surg 1998;155:476-80.

3 Noguchi Y, Imada T, Matsumoto A, et al. Radical surgery for gastric cancer: a review of Japanese experience. Cancer 1989;64:2053-62.

4 Sano T, Kobori O, Muto T. Lymph node metastasis from early gastric cancer: endoscopic resection of tumor. $\mathrm{Br} \mathcal{F}$ Surg 1992;79:241-44.

5 Yamao T, Shirao K, Ono $\mathrm{H}$, et al. Risk factors for lymph cer 1996;77:602-6.

6 Tada M, Mukrakami A, Karita M, et al. Endoscopic resection of early gastric cancer. Endoscopy 1993;25:44551.

7 Sano T, Okuyama Y, Kobori O, et al. Early gastric cancer; endoscopic diagnosis of depth of invasion. Dig Dis Sci 1990;35:1340-44.

8 Tada M, Shimada M, Murakami F. Development of the strip-off biopsy. Gastroenterol Endosc 1984;26:833-9.

9 Gotoda T, Kondo H, Ono H, et al. A new endoscopic mucosal resection procedure using an insulation-tipped diathermic knife for rectal flat lesions. Gastrointestinal diathermic knife for recta
Endosc 1999;50:560-63.

10 Japanese Gastric Cancer Association. Japanese classification of gastric carcinoma, 2nd English edn. Gastric Cancer 1998;1:10-24.
1 Nishi M, Omori Y, Miwa K, eds. Japanese Research Society for Gastric Cancer. Fapanese Classification of Gastric node metastasis from intramucosal gastric carcinoma. Can- 
11 Schlemper RJ, Itabashi M, Kato Y, et al. Differences in diagnosis criteria for gastric carcinoma between Japanese and Western pathologists. Lancet 1997;349:1725-9.

12 Everett SM, Axon ATR. Early gastric cancer in Europe. Gut 1997;41:142-50.

13 Yasuda K, Shiraishi N, Suemasu T, et al. Rate of detection of lymph node metastasis is correlated with the depth of submucosal invasion in early gastric cancer. Cancer 1999;85: 2119-23.
14 Inoue $\mathrm{H}$, Tani $\mathrm{M}$, Nagai $\mathrm{K}$, et al. Treatment of esophageal and gastric tumors. Endoscopy 1999;31:47-55.

15 Hirao M, Matsuda K, Asanuma T, et al. Endoscopic resection of early gastric cancer and other tumors with local injection of hypertonic saline-epinephrine. Gastrointest Endosc 1998;34:264-9.

16 Oda I, Kondo H, Gotoda T, et al. Endoscopic diagnosis for multiple early gastric cancer [abstract]. Proceeding of the $3 \mathrm{rd}$ International Gastric Cancer Congress, 1999:343;AO4069. 